\title{
A study on clinical manifestations and outcome of tuberculous meningitis among children with reference to BCG vaccination status and nutritional status
}

\author{
Srinivasa $\mathbf{A}^{1}$, Gayakwad V.S. ${ }^{2}$ \\ ${ }^{1}$ Dr. Srinivasa Arer, Department of Pediatrics, Gadag Institute of Medical Sciences, Gadag, Karnataka, ${ }^{2}$ Dr. Vasundara S. \\ Gayakwad, Department of Community Medicine, Sri B.M. Patil Medical College Bijapur, Karnataka, India.
}

Corresponding Author: Dr. Vasundara S Gayakwad C/o Dr. Srinivasa Arer, H. No. 14/156B Kalasapur Road, Adarsh Nagar, Gadag, Karnataka, India. E-mail: vasundara.gayakwad@gmail.com

\begin{abstract}
Background: Childhood tuberculosis is largely underestimated due to diagnostic difficulties and non-specific clinical manifestations, even in many tertiary care hospitals. With increase in coverage of BCG vaccination and improved nutritional status in children, modified clinical pictures of childhood tuberculous meningitis (TBM) has been observed in some research studies. Objectives: To study the different clinical manifestations and outcome of TBM in relation to BCG vaccinated, non- vaccinated and nutritional status of the children, in VIMS Bellary. Methodology: This is a case series prospective study. Patients admitted in the paediatric emergency ward from Nov 2003 to Nov 2004 were recruited for the study. The patients were classified according to their immunization status and nutritional status. Results: TBM constituted $29 \%$ of the total admitted TB cases. Majority were below 5 years. Fever, vomiting, positive McEwan's sign and meningeal irritation signs were the commonest features observed. Atypical features were present in one third of the cases. No significant differences were seen between vaccinated and non-vaccinated patients. Significant differences, however, were observed in the clinical features and mortality between severely malnourished and well-nourished TBM cases in this study. Conclusion: It was concluded that clinical patterns in TBM vary according to nutritional status of the child and severe malnutrition carry very poor prognosis. Knowing different clinical patterns of the disease helps in early prognosis and thus in preventing deaths due to TBM among children.
\end{abstract}

Key words- Tuberculous Meningitis, BCG immunisation status, Severe Malnutrition

\section{Introduction}

The World health organization (WHO) estimates that one third of the world's population is infected with mycobacterium tuberculosis, with the highest prevalence of tuberculosis in Asia [1]. The prevalence of childhood tuberculosis is largely underestimated, usually as a result of diagnostic problems. Clinical manifestations like lethargy, fever, vomiting, weight loss, altered mental status, photophobia being nonspecific particularly in the early stages of the disease, tuberculosis remains easily misdiagnosed, under diagnosed or paradoxically over treated. Only 30$40 \%$ childhood cases are confirmed even in tertiary care institutions [2]. Younger children also experience more severe disease such as meningitis and disseminated disease [3].

Manuscript received: $10^{\text {th }}$ June 2019

Reviewed: $20^{\text {th }}$ June 2019

Author Corrected: $28^{\text {th }}$ June 2019

Accepted for Publication: $3^{\text {rd }}$ July 2019
Tuberculous Meningitis (TBM), the most dangerous form of extra-pulmonary tuberculosis, occurs in 7-12\% of tuberculosis patients in our country and has high fatality rate despite availability of effective chemotherapy. Fatality rates and neurological sequelae depend on many factors, including age of the patient, nutritional status, time of diagnosis, stage of disease at presentation and use of appropriate anti-tubercular therapy.

The protective efficacy of BCG has ranged from $0-80 \%$ in various trials. Nothing is more controversial than passing judgment on the efficacy of BCG vaccine $[4,5]$. BCG vaccination is known to stop the haematogenous spread of tuberculous disease thus leading to localization of the disease. With increased coverage of vaccination in children, what we see today in BCG vaccinated and well-nourished children, are the modified clinical pictures of neurotuberculosis, 


\section{Original Research Article}

specially serous tuberculous Meningitis, localized tubercular disease in meanings and brain, with wide varieties of clinical presentations depending upon the site of the lesion $[6,7]$.

Also, the results of BCG vaccination in malnourished children are often disappointing in our country [6]. Classical or generalized TBM, military TB, disseminated TB and other serious complications of primary infection go on occurring in malnourished BCG vaccinated children $[6,8]$. Hence it is useful to relearn the new patterns of tuberculous disease if any, in relation to vaccination status and nutritional status of the children.

The published data regarding, the various clinical forms and outcome of TBM and relation to BCG vaccination status are limited [7]. Also wide availability of CT scan and neurosonogram has contributed to a better understanding of the clinical features of TBM and its management. Hence a study of various clinical features and outcome of TBM was undertaken and tried to find any differences between vaccinated and non-vaccinated children. Any difference between well-nourished and poorly nourished is also noted.

\section{Aims and Objectives}

1. To study the different clinical manifestations and outcome of TBM in BCG vaccinated and nonvaccinated children, admitted in a government teaching hospital.

2. To study the different clinical manifestations and outcome of TBM in relation to nutritional status of the children.

\section{Materials and Methods}

Study design: Case series prospective study

Setting: Pediatric emergency ward, VIMS, teaching Hospital, Bellary

Participants: Forty Eight children with definite or probable tuberculous meningitis (TBM)

Ethical Approval: Permission for the study was obtained from the College authorities prior to commencement

Duration of the study was Nov 2013 Nov 2014. Local cultural values and ideas were respected. Confidentiality was assured. The nature and purpose of the survey were explained in detail to them in their own language.

\section{Method of data Collection}

a) This is a prospective study of series of consecutive patients diagnosed as having TBM from NOV 2003 to Nov 2004.

b) All studies were performed after obtaining informed consent in patient's native language.

c) All these individuals were subjected to detailed clinical and neurological examination and various laboratory tests, including complete Haemogram, ESR, Mantoux test, biochemistry, LP for CSF Analysis, Transcranial neurosonogram / CT scan brain or both in majority of cases for the evidence of chronic meningitis changes and its complications.

d) Patients were considered as immunized with BCG if there is a clear BCG scar on the left deltoid region.

\section{Inclusion criteria/case definition}

1. Patients who satisfy clinical case definition of TBM, which includes history, clinical features, fundoscopy, etc.

2. Demonstration of acid-fast bacilli in the CSF or fulfillment of the following criteria.

A. Essential: CSF showing:
a. Predominant lymphocyte pleocytosis $>50 / \mathrm{mm}^{3}$
b. Protein $>60 \mathrm{mg} \%$
c. Sugar $<2 / 3$ of blood sugar

\section{B. Supportive}

Along with essential ones, two or more of the following clinico-investigational criteria must be present:

a. History of fever of two weeks or more

b. Positive family history of tuberculosis

c. Generalised lymphadenopathy

d. Mantoux test (5TU) $>10 \mathrm{~mm}$

e. Positive radiological evidence of tuberculosis elsewhere in the body

f. Isolation of AFB from gastric lavage or other sites

g. CT scan evidence of basal exudates or CNS tuberculosis

h. Histologically proven tubercular lymphadenitis

Nutritional status of the children is assessed according to IAP classification i.e., Nutritional subcommittee of the Indian Academy of Paediatrics (standard value).

Exclusion criteria: A child with any neurological symptom with proven etiology like CSF positive for specific etiology like pyogenic meningitis, positive for $\mathrm{J}$ E virus, malaria parasite etc. 


\section{Original Research Article}

Statistical Methods: Descriptive statistics like mean, percentages were used. Chi square test was applied to find out the statistical significance of TBM in relation to BCG vaccination and different grades of nutrition. $P$ value of $<0.05$ was considered statistically significant and $<0.001$ as highly significant.

Clinical data: The neurological status of all patients with TBM was evaluated on admission and during follow up and the clinical manifestations were classified as follows

\section{Classical TBM}

Stage I: Prodromal stage: No definite neurological symptoms at admission or in the history before admission.

Stage II: Stage of meningeal irritation: meningitis signs, drowsiness or lethargy, cranial nerve palsies.

Stage III: Stage of diffuse or focal cerebral involvement: severe clouding of consciousness, stupor or coma, convulsions, gross paresis or paralysis.

Modified forms of TBM: Any isolated /unusual CNS feature with or without global encephalopathy

a. Serous tubercular meningitis.

b. Isolated tuberculous encephalopathy. c. Multiple intracranial tuberculomas (meningeal).

d. Localised lesions manifestations due to infarctions in the territory of large intracranial arteries and / or small arteries with lacunar infarct with resultant large number of syndromes.

e. Localised meningitis in the posterior fossa.

f. Isolated spinal tuberculous meningitis.

\section{Therapy and outcome:}

Therapy: All patients received ATT (anti-tubercular treatment) according to body weight. $2 \mathrm{HRZE}+10 \mathrm{HRE}$ regimen was followed.

In addition children received steroid for 10-12 weeks. Anti-edema and other measures were considered whenever indicated.

Outcome: At discharge and at 3 months follow up outcome was graded into 3 groups according to modified British medical research council clinical criteria.

Outcome I = complete recovery.

Outcome II = partial recovery with residual deficits/disability.

Outcome III = Death.

\section{Results}

Mean age of study subjects was 3.4 years with range between 6months to 12 years. Majority were in the age group of 1-5 years $(64.5 \%)$ followed by infants $(16.7 \%)$. The male to female ratio was $1: 1$.

Mean duration of illness was about 12.6 days prior to establishment of diagnosis at our hospital. The mean duration of stay of the cases at our hospital was 18.1 days. Majority of the study subjects belonged to low socio economic group $(81 \%)$.

According to BCG immunization status, 58\% (28) were immunized and 42\% (20) were unimmunized. With respect to nutritional status, 33.3\% (16) had grade III malnutrition followed by $25 \%$ (12) grade IV, 25\% (12) grade II, 12.5\% (6) grade I malnutrition. 4.7\% (2) children had normal nutritional status and all of them were immunized with BCG. All unimmunized children had one or the other grades of malnutrition.

Symptoms: Fever was the most common symptom in 45 cases (94\%) followed by vomiting and altered sensorium in 31 cases (65\%). 25 cases presented with convulsions, 60\% generalized tonic clonic and 40\% complex partial type.

Headache was present in $26(54 \%)$ of cases. The other symptoms were cough $(38 \%)$, refusal of feeds $(11 \%)$, weakness of limbs $(41.6 \%)$, ear discharge $(10 \%)$, diarrhoea $(3 \%)$, deviation of mouth $(3 \%)$ etc.

Signs: The most common sign elicited at the time of admission was McEwan's sign in 37 cases (77\%), signs of meningeal irritation (neck rigidity, Kernig's sign, Brudzinski’s sign) was present in $61 \%$ of the cases. Any focal deficits, with global encephalopathy are considered as classical TBM. 


\section{Original Research Article}

Hemiparesis, with global encephalopathy was present in $48 \%$, cranial nerve palsies were present in $52 \%$ cases out of which 10 cases had $7^{\text {th }} \mathrm{CN}$ palsy, 8 had $3^{\text {rd }} \mathrm{CN}$ palsy and 7 had combined $3^{\text {rd }}, 6^{\text {th }}$ and $7^{\text {th }} \mathrm{CN}$ palsy. Fundal changes were seen in 14 cases $(29 \%)$, most of them showing papilledema. Only 2 cases had optic atrophy. No choroid tubercle was seen in this study. 22 cases $(46 \%)$ came with decreased sensorium, Glasgow Coma scale (GCS) between 10-14.24 cases (15\%) had GCS less than 10 at the time of admission. 2 cases fully conscious. 7 cases had decerebrate rigidity and 3 cases had decorticated rigidity.

Atypical presentations: Following atypical presentations of TBM (without diffuse involvement of meninges/global encephalopathy) were observed in 16 (33\%) of the cases. More than one atypical presentation has been observed in some patients. The most common feature was meningeal tuberculoma (6 cases) followed by localised or focal areas of meningitis (5 cases) and encephalitis. Table 1

Table-1: Clinical Profile of tuberculous meningitis among study subjects.

\begin{tabular}{|c|l|c|}
\hline SI. No. & Clinical characteristics & TBM cases $\mathbf{N}=\mathbf{4 8}(\mathbf{\%})$ \\
\hline I & Classical symptoms & \\
& Fever & $45(93.8)$ \\
& Convulsions & $27(56.3)$ \\
& Vomiting & $30(62.5)$ \\
& Altered Sensorium & $30(62.5)$ \\
& Cough & $17(35.4)$ \\
& Headache & $27(56.3)$ \\
\hline II & Classical signs & \\
& Meningeal irritation & $29(60.5)$ \\
& Hemiparesis & $23(47.9)$ \\
& Cranial nerve palsy & $25(52.1)$ \\
& Fundal changes & $13(27.1)$ \\
& McEwan's sign & $36(75)$ \\
& GCS =15 & $2(42)$ \\
& 10-14 & $21(43.7)$ \\
& $<10$ & $25(52.1)$ \\
\hline III & Atypical Features & \\
& Isolated ophthalmoplegia & $3(6.2)$ \\
& Brain stem syndromes & $2(4.7)$ \\
& Serous meningitis & $2(4.7)$ \\
& Localised meningitis/edema(CT scan) & $5(10.4)$ \\
& Conscious type & $3(6.2)$ \\
& Encephalopathy (CT scan) & $2(4.7)$ \\
& Meningeal tuberculoma (CT scan) & $6(12.5)$ \\
& Focal infarcts (CT scan) & $4(8.3)$ \\
\hline
\end{tabular}

Other typical features found were Ophthalmoplegia (3), brain stem syndromes (2), serous meningitis (2), conscious type (3), encephalopathy (2) and focal infarcts in 4 cases.

S/S in BCG vaccinated: The common symptoms observed were fever $93 \%$, altered sensorium $64 \%$, vomiting $54 \%$ and convulsions $50 \%$. Other symptoms observed were cough, headache. The common signs present were GCS below 10 in $57 \%$ of cases, meningeal irritation in $64 \%$, and McEvan's sign in $60 \%$. Also seen were hemiparesis $43 \%$, CN palsy $43 \%$, fundal changes $25 \%$. All signs were significantly more common in malnourished children except GCS10-14. Tuberculomas, conscious variety and focal involvements are seen more commonly in well-nourished group among vaccinated patients. All atypical features of TBM are more common in well-nourished group when compared to malnourished children except encephalopathy. 


\section{Original Research Article}

In BCG negative children also, fever was found in majority of the cases (95\%). This was followed by vomiting $75 \%$, convulsions $65 \%$, altered sensorium $60 \%$ and headache $50 \%$. Cough was seen in $25 \%$ of patients. Meningeal irritation was present in 55\%, McEwan's sign in 95\%, hemiplegia in 55\%, CN palsy in $65 \%$, fundal changes in $30 \%$, GCS below 10 in $45 \%$ of cases. Majority of the signs were significantly more common in poorly nourished children.

Focal involvement $15 \%$, brainstem syndromes $20 \%$, tuberculomas were commonly observed features. Other features were ophthalmoplegia, encephalopathy and focal infarcts.

Among the vaccinated patients, 57\% had classical disease and 43\% had atypical disease. In non-vaccinated group 40\% had classical disease and $60 \%$ had atypical disease.This difference was not statistically significant.(p value $>0.05)$ Table 2

Table-2: Classical and Atypical Features of TBM in Relation to Vaccination Status

\begin{tabular}{|c|c|c|c|}
\hline Immunization status & Classical & Atypical & Total \\
\hline BCG +ive & $16(57.1)$ & $12(42.9)$ & 28 \\
\hline BCG -ive & $8(40)$ & $12(60)$ & 20 \\
\hline Total & $\mathbf{2 4}(\mathbf{5 0})$ & $\mathbf{2 4}(\mathbf{5 0})$ & $\mathbf{4 8}$ \\
\hline
\end{tabular}

Among the well-nourished patients, 20\% had classical disease and 80\% had atypical disease. In severely malnourished children $85.7 \%$ had classical disease and $14.3 \%$ had atypical disease. This difference was statistically significant. (P value $<0.05)$ Table 3

Table-3: Classical and atypical Features of TBM in relation to nutritional status.

\begin{tabular}{|c|c|c|c|}
\hline Nutritional status & Classical & Atypical & Total \\
\hline Well nourished & $4(20)$ & $16(80)$ & 20 \\
\hline Poorly nourished & $24(85.7)$ & $4(14.3)$ & 28 \\
\hline Total & $\mathbf{2 8}(\mathbf{5 8 . 3})$ & $\mathbf{2 0}(\mathbf{4 1 . 7 )}$ & $\mathbf{4 8}$ \\
\hline
\end{tabular}

In BCG +ive normally nourished group 33\% presented in grade I disease with full recovery (outcome1). 67\% presented in grade II disease of which $43 \%$ recovered completely, $43 \%$ partially recovered and $14 \%$ died. None were in grade III disease.

In poorly nourished children, none had grade I disease , 5 had grade II disease in which 2 recovered partially, 1 recovered fully and 1 died.11 patients had grade III disease, one patient was referred to higher centre, 4 patients recovered partially and 6 patients died. Table 4

Table-4: Outcome of TBM among BCG vaccinated children in relation with nutritional status.

\begin{tabular}{|c|c|c|c|c|c|}
\hline Sl. No & Stage & Outcome 1 n (\%) & Outcome 2 n (\%) & Outcome 3 n (\%) & Total \\
\hline \multicolumn{7}{|c|}{ Well nourished } \\
\hline 1 & Stage1 & $4(100)$ & $0(0)$ & $0(0)$ & 4 \\
\hline 2 & Stage2 & $4(50)$ & $3(38.5)$ & $0(0)$ & 0 \\
\hline 3 & Stage3 & $0(0)$ & $0(0)$ & $0(0)$ & 0 \\
\hline \multicolumn{7}{|c|}{ Malnourished } \\
\hline 4 & Stage1 & $0(0)$ & $0(0)$ & $1(20)$ & 5 \\
\hline 5 & Stage2 & $1(20)$ & $3(60)$ & $6(54)$ & $10^{*}$ \\
\hline 6 & Stage3 & $0(0)$ & $4(36)$ & & \\
\hline
\end{tabular}

(*11-1= 10 as one stage 3 diseased patient was referred to higher centre) 


\section{Original Research Article}

Among the BCG -ive normally nourished patients, 1 patient was in grade I disease and recovered fully. 7 (35\%) patients were in grade II, 4 patients recovered fully, 3 partially and none died. Only one patient was in grade III disease which recovered partially. Among the malnourished patients, none had grade I meningitis. Only 1 was in grade 2 which recovered partially. Majority, $10(50 \%)$ were in grade III disease among which one went against medical advice, one referred to higher centre. In the remaining 8 patients half recovered partially and half died. Table 5

Table-5: Outcome of TBM among BCG non-vaccinated children $(n=20)$ in relation with nutritional status

\begin{tabular}{|c|c|c|c|c|c|}
\hline Sl. No & Stage & Outcome 1 n (\%) & Outcome 2 n (\%) & Outcome 3 n (\%) & Total \\
\hline \multicolumn{7}{|c|}{ Well nourished } \\
\hline 1 & Stage1 & $1(100)$ & $0(0)$ & $0(0)$ & 0 \\
\hline 2 & Stage2 & $4(67)$ & $2(33)$ & $0(0)$ & 1 \\
\hline 3 & Stage3 & $0(0)$ & $1(100)$ & $0(0)$ & 0 \\
\hline \multicolumn{7}{|c|}{ Malnourished } & $0(0)$ & 2 \\
\hline 4 & Stage1 & $0(0)$ & $2(100)$ & $4(50)$ & $8^{*}$ \\
\hline 5
\end{tabular}

$(* 10-2=8$ as two stage 3 diseased patients were referred to higher centre)

Sequel at the time of discharge- Totally 18 patients survived with sequel out of 45 patients, most of them were from grade II and grade III disease. The sequel observed at the time of discharge and follow up were seizures $17 \%$, mental retardation in 39\%, optic atrophy in 5\% and obesity in $11 \%$, hemiplegia and other focal deficits were present in $33 \%$.

\section{Discussion}

Tuberculosis continues to be major health problems of the developing countries and TBM continues to be its most dreaded complication. TB accounted for 161 admissions out of 4774 cases admitted to this hospital during the study period. This constituted $3 \%$ of all admissions. TBM constituted $1 \%$ of the total cases during this study period. This is lower when compared to other studies done by Udani et al (1.9\%), Benakappa et al $(2 \%)$ and Chandrashekar et al $(1 \%)[10,11,12]$. Among the TB admissions TBM constituted $29.8 \%$ in the present study. Udani et al (29\%) study findings were consistent with the present study whereas Benkappa et al (47.4\%) and Chandrashekar et al (18.6\%) studies showed contrast findings $[10,12]$.

$81 \%$ of cases in the present study were below 5 years of age depicting that majority of cases occurring in less than 5 years children. As compared to present study slightly higher incidence was found in Benakappa et al [11] study and slightly lower incidence was found in Udani et al and Brijendra Garg et al studies [10, 13]. Gender distribution was found to be equal in his study whereas in other studies have shown a slight male preponderance. Male preponderance could be due to more outdoor exposure to TB patients and preference given by parents in seeking health care.
In the present study, $25 \%$ gave history of contact with TB which was higher compared to Benkappa et al (16\%) and lower compared to Kennedy (56\%) [11].

More than half $(58 \%)$ of the children were immunized in the present study. As compared to the present study immunization status was found to be lower in degefie $\mathrm{T}$ study (33\%) [15].

In the present study, majority (58\%) had severe malnutrition (grade III and Grade IV) and 38\% had mild to moderate malnutrition (grade I and Grade II). The high incidence of PEM could be because of low socioeconomic status, rural and agricultural background.

In the present study fever was the most common symptom followed by altered mental status and vomiting. The variations in symptoms could be because history is indirect as perceived by parents and other family members rather than directly told by patient. Similar to the present study other studies also found fever as one of the most common symptom (Ramachandra, Benakappa, Udani peter décor) $[16,11$, 10]. In contrast, other studies found convulsion as next most common symptom followed by altered sensorium 


\section{Original Research Article}

and vomiting. Other symptoms in the present study were cough and headache which were higher compared to Ramachandra et al study [16]. Positive McEwan's sign/tense anterior fontanels and signs of meningeal irritation were the commonest signs. Similar findings were found in other studies $[10,11]$. Signs of meningeal irritation were lower in the present study as compared to other studies whereas McEwan's sign/tense anterior fontanel was higher.

Atypical clinical features were present in 33\% cases, most common being meningeal tuberculomas (one third cases) followed by local meningitis. Present study reported that mean duration of illness before seeking medical advice was 12.6 days. In contrast, degefie $\mathrm{T}$ and Kondo $\mathrm{S}$ studies reported longer mean duration of illness [15,16]. In the present study no statistically significant difference in clinical manifestations was seen between vaccinated and non-vaccinated children. Guler N. [18] Ones U series made similar conclusion. Udani et al in their study have reported difference in manifestations between the 2 groups [10].

Significant difference was observed in clinical features like neck rigidity, cranial nerve deficits, focal neurological signs between well-nourished and severely malnourished children in this study. No comparative studies were available for the same although Udani et al and Awasthi series proposed similar opinions [10,7].

Case fatality rate was $27 \%$. There were no deaths in grade I disease, $9.5 \%$ died in grade II and $53 \%$ in grade III. All the survivors of grade III disease had residual disability. This was lower compared to Girgi"s NI et al (57\%), [19] Al abbasi [20] (42\%) and higher compared to Zafar Iqbal et al (23\%) [21]. Among various factors, coma or stage III was found to be most significantly associated with poor outcome and mortality in the present study. Findings of studies done by Zafer et al, Kalita et al, Girgi"s NI et al were consistent with the present study [21, 22, 19].

There was no significant difference in mortality between vaccinated (29\%) and non-vaccinated children $(22 \%)$. This could be due to poor immunity, secondary to malnutrition and poor living conditions. Al Abbasil reported lower case fatality rate in BCG vaccinated (13\%) than non-vaccinated children (26\%) [20].

The mortality was significantly high in severely malnourished children (44\%) compared to wellnourished children (5\%). Similar findings were found in Gupta S study [23].
Limitations: as this is a case series study, associated factors could have been better studied by case control study.

\section{Conclusion}

Tuberculous meningitis (TBM) is an important cause of admission in children with tuberculosis in the present study area. The disease can present in many different forms. The clinical features and outcome of TBM may vary between well-nourished and severely malnourished children. There appears to be no difference in clinical features and outcome of disease in vaccinated and nonvaccinated children.

Further studies which includes other factors that influence the disease pattern need to be studied.

This study stresses the importance of good nutrition for a better outcome in TBM patients.

\section{Contribution details}

Dr. Srinivasa Arer handled the concept, design, literature search, data acquisition, data analysis, statistical analysis, manuscript preparation, editing, and review.

Dr. Vasundara S Gayakwad contributed through the handling of concept, design, manuscript editing, review and guarantor.

Acknowledgements: Authors would like to express my profound gratitude to all the participants. I also acknowledge Dr Veerashankar M. Head of Department, department of pediatrics for guiding this project.

Funding: Nil, Conflict of interest: None initiated, Perission from IRB: Yes

\section{References}

1. Ahmadinejad Z, Ziaee V, Aghsaeifar M, Reiskarami SR. The prognostic factors of tuberculous meningitis. Int J Infect Dis. 2003;3(1).153-4.

2. Mahadevan S. Advances in diagnosis of tuberculosis. Indian J Pediatr. 1996;63(2):153-7.

3. Donald PR. Children and tuberculosis: protecting the next generation? Lancet. 1999; 353 (9157): 1001-2. DOI:10. 1016/s0140-6736(99)02010-3

4. Udani PM. BCG vaccination in India and tuberculosis in children: newer facets. The Indian Journal of Pediatrics. 1994;61(5):451-62. 


\section{Original Research Article}

5. Dastur DK, Manghani DK, Udani PM. Pathology and pathogenetic mechanisms in neurotuberculosis. Radio Clin North Am. 1995;33(4):733-52.

6.Awasthi S, Moin S. Effectiveness of BCG vaccination against tuberculous meningitis. Indian Pediatr. 1999;36 (5):455-60.

7. Seth V, Gulati S. clinical manifestations, Diagnosis and treatment In; Essentials of tuberculosis in children Ed. Set V. New Delhi; Jaypee Brothers 2003:134-62.

8. Peter D Corr. Imaging in CNS Tuberculosis Available at www.e-medicine.com/radio/topic 720 .

9. Bernaerts A, Vanhoenacker FM, Parizel PM, Van Goethem JW, Van Altena R, Laridon A, et al. Tuberculosis of the central nervous system: overview of neuroradiological findings. Eur Radiol. 2003;13(8): 1876-90. Epub 2002. DOI:10.1007/s00330-002-1608-7

10. Benakappa DG, Chandrasekhar SK, Chandrasekhar P, Kakkur M, Bhargava KM. Tuberculous meningitis: review of 50 cases. Ind Pediat. 1975;12(11):1161.

11. Chandrashekar- Clinical profile and outcome of tuberculous meningitis in children at district hospital, Bellary. thesis submitted to department of paediatrics VIMS Bellary 1999.

12. Garg BK. Tuberculous meningitis in children. Ind $\mathbf{J}$ Paediat 1981;44(3):87.

13. Kennedy DH, Fallon RJ. Tuberculous meningitis. JAMA. 1979;241(3):264-8.

14. Degefie T. Tuberculous meningitis in a district hospital from Southern Ethiopia. Ethiop Med J. 2003;41 (4): $311-8$.
15. Ramachandran RS, Ramanathan K, Indra G. Tuberculous meningitis. A review of 288 cases in children. Indian J Pediatr. 1970;37(266):85-8.

16. Kondo S, ITO M. Usefulness of cranial and chest imaging in the diagnosis of tuberculous meningitis among infants and young children. Kekkaku (Tuberculosis). 2003; 78(2):89-93.

17. Girgis NI, Sultan Y, Farid Z, Mansour MM, Erian MW, Hanna LS et al. The effect of prior BCG vaccination on the clinical and radiographic presentation of tuberculosis meningitis in children in Istanbul, Turkey. Int J Tuberc Lung Dis. 1998;2(11): 885-90.

18. Girgis NI, Sultan Y, Farid Z, Mansour MM, Erian MW, Hanna LS. et al. Tuberculosis meningitis, Abbassia Fever Hospital-Naval Medical Research Unit No. 3-Cairo, Egypt, from 1976 to 1996. Am J Trop Med Hyg. 1998; 58 (1): 28-34. DOI: 10.4269/ajtmh. 1998. 58.28

19. Al-Abasil AM. Tuberculous meningoencephalitis in Baghdad,1993-99: a clinical study of 224 cases. Eastern Health J. 2002;8(2):3.

20. Malik ZI, Ishtiaq O, Shah NH, Anwer F, Baqai HZ. Analysis and outcome of 30 patients with tuberculous meningitis. Pak J Med Res. 2002;41(4):137-41.

21. Kalita J, Misra UK. Outcome of tuberculous meningitis at 6 and 12 months: a multiple regression analysis. Int J Tuberc Lung Dis. 1999;3(3):261-5.

22. Gupta S, Chopra K. Tuberculous meningitis in children. Ind. J Tub. 1981;28(1):261-5.

\section{How to cite this article?}

Srinivasa A, Gayakwad V.S. A study on clinical manifestations and outcome of tuberculous meningitis among children with reference to BCG vaccination status and nutritional status. Int J Pediatr Res. 2019;6(07):310-317.doi:10. 17511/ijpr. 2019.i07.01 